\title{
Análisis filogenético de especies de Quercus L. utilizando tres códigos de barras de ADN
}

\section{Phylogenetic analysis of Quercus L. species with three DNA barcode genetic markers}

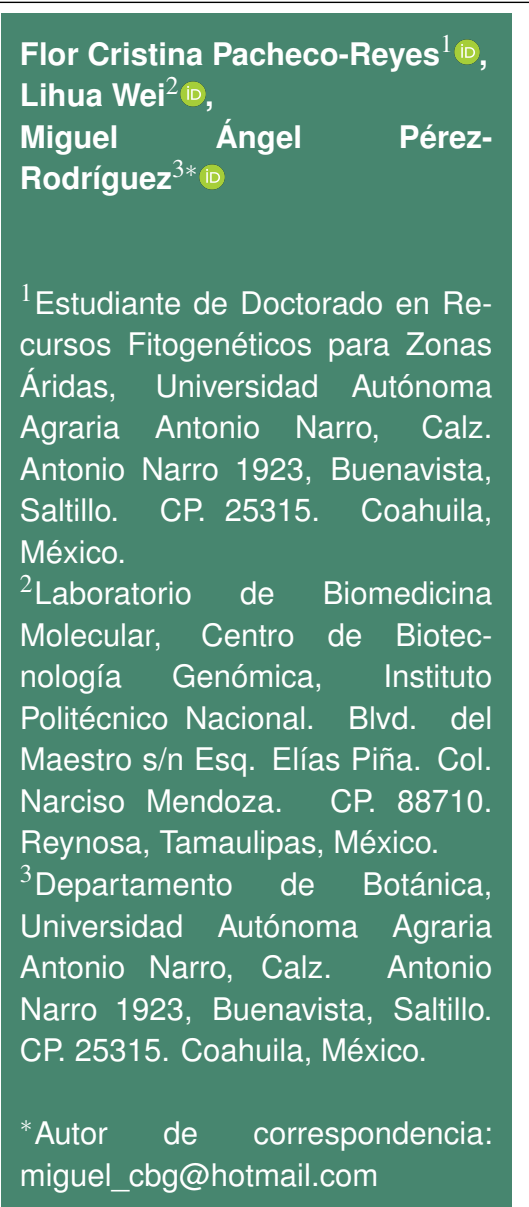

Artículo científico

Recibido: 01 de febrero 2021

Aceptado: 09 de mayo 2021

Como citar: Pacheco-Reyes FC, Wei L, Pérez-Rodríguez MA (2021) Análisis filogenético de especies de Quercus L. utilizando tres códigos de barras de ADN. Ecosistemas y Recursos Agropecuarios 8(2): e2831. DOI: $10.19136 /$ era.a8n2.2831
RESUMEN. El género Quercus L., Fagaceae, es de gran relevancia en cuanto diversidad, importancia ecológica y económica; pero la información taxonómica documentada no siempre permite distinguir entre los taxones. En este estudio se evaluó el poder discriminatorio de tres regiones de ADN (rbcL, matK e ITS2) como códigos de barras para identificar especies del género Quercus. Los datos analizados corresponden a 56 secuencias de rbcL, 51 de ITS2 y 61 de matK, provenientes de 92 especies (11 mexicanas y 81 de Eurasia y América del Norte). Los cebadores universales (rbcL e ITS2) amplificaron el $100 \%$ de las muestras de encinos mexicanos. El análisis BLAST de los fragmentos rbcL e ITS2 de encinos mexicanos, proporcionó un poder de identificación alto con 98.1 y $98.3 \%$, respectivamente. Con el método basado en la distancia genética se tuvieron tasas de discriminación extremadamente bajas, posiblemente debido a distancias genéticas superpuestas, además de la frecuente hibridación entre especies y la baja tasa de variación. El método de unión de vecinos cercanos proporcionó un poder discriminatorio alto con los marcadores matK (81.96\%) e ITS2 (80.39\%), generando agrupaciones por regiones geográficas y por secciones del subgénero Quercus en los arboles filogenéticos. Según el rendimiento general de dicho método se propone el uso de ITS2 y matK como los códigos de barras de ADN más adecuados para la identificación de especies del género Quercus.

Palabras clave: Discriminatorio, encino, ITS2, matK, rbcL.

ABSTRACT. The genus, Quercus L, Fagaceae has great relevance in terms of diversity, ecological and economic importance. However, the recorded taxonomic information does not always distinguish the classification. In this study, three DNA regions (rbcL, matK and ITS2) were used as barcodes to identify species of the genus Quercus. The analyzed data (56 rbcl, 51 ITS and 61 matK sequences) corresponded to 92 species (11 are from Mexican, and 81 are from Eurasia and North America). The universal primers (rbcL and ITS2) amplified $100 \%$ of Mexican oak samples. The BLAST analysis of the rbcL and ITS2 fragments of Mexican oaks, showed a high identification ability with 98.1 and $98.3 \%$ respectively. Genetic distance-based method resulted in extremely low discrimination rates, possibly due to overlapping genetic distances, as well as frequent inter-species hybridization and a low rate of variation. The neighbor-joining method provided a high discriminatory ability with matK $(81.96 \%)$ and ITS2 (80.39\%) markers, generating groupings based on geographic regions and sections of the Quercus subgenus in phylogenetic trees. According to the overall performance, ITS2 and matK are recommended to use as the most suitable DNA barcodes to identify Quercus species.

Key words: Discriminatory, oak, ITS2, matK, rbcL. 


\section{INTRODUCCIÓN}

El género Quercus L., es el grupo más grande de la familia Fagaceae, presenta la mayor distribución y diversidad a nivel mundial, con unas 500 especies aceptadas (Valencia et al. 2016). México es considerado el centro de diversificación más grande a nivel mundial, estas especies se distribuyen en regiones montañosas del centro y noreste del país principalmente (Ramamoorthy et al. 1993, Govaerts y Frodin 1998). En México se han identificado 161 especies del género Quercus que pertenecen al subgénero Quercus, con tres secciones: Quercus, Lobatae y Protobalanus (Valencia 2004). Existe gran interés en torno a este grupo taxonómico, debido a su alta diversidad y su importancia ecológica y económica (Romero-Rangel 2006, Gual-Díaz 2014, Pérez-Mojica 2017). Debido a la variación taxonómica y morfológica dentro de la misma especie, al largo de tiempo de generación y la hibridación frecuente entre especies, se dificulta conocer el número real de especies (Villarreal et al. 2008, Sabás et al. 2017). Por lo anterior, es necesario proporcionar una solución a los problemas taxonómicos, que permita una mejor toma de decisiones para el manejo y conservación de estas especies (Valencia 2004, Hoban et al. 2009, Hipp et al. 2013). Por tal motivo, los códigos de barras de ADN proporcionan datos que ayudan a discernir relaciones taxonómicas y permiten una mejor distinción en especies con alto grado de similitud a niveles de detalles altamente específicos (Borek y Summer 2009, Govindaraghavan et al. 2012).

En el 2009 el Consorcio del Código de Barras de $A D N$ (CBOL) recomendó en plantas el uso de fragmentos provenientes del genoma del cloroplasto, tales como rbcL (ribulosa-1-5 bifosfato carboxilasa/oxigenasa) y matK (maturasa $\mathrm{K}$ ), los cuales son relativamente fáciles de amplificar debido a su estabilidad relativa, son de copia única, estructura altamente conservada y herencia uniparental (China Plant BOL Group et al. 2011). En 2011 se sugirió el uso de la región nuclear ITS2 (espaciador interno transcrito) como complemento, esta región pertenece a los genes que codifican para el ARN ri- bosómico (CBOL Plant Working Group et al. 2009). La información que proporcionan los códigos de barra de ADN es utilizada con diferentes propósitos, entre ellos: hacer inventarios de biodiversidad, reconstrucción del clima y la vegetación del pasado, además, sirve como complemento de la filogenética, ya que con la integración de datos moleculares y homologías morfológicas se logra la obtención de información sobre la historia evolutiva de las especies (Hajibabaei et al. 2007, Kress et al. 2009, Costion et al. 2011). Al respecto, Hipp et al. (2013) afirmaron que es necesario establecer un método preciso, específico y universal que proporcione datos confiables para la identificación de especies y provea información sobre patrones filogenéticos en especies de Quercus. En México, actualmente sólo el 5\% del total de las especies de Quercus distribuidas en el país cuentan al menos con uno de los tres códigos, lo cual representa una limitante para hacer análisis a mayor escala, y muestra la necesidad de realizar más estudios con la tecnología de código de barras de ADN con especies de distribución nacional. Por lo anterior, el objetivo de este trabajo fue determinar las relaciones filogenéticas en especies de encinos de tres regiones específicas de México, Estados Unidos y Eurasia, para demostrar el poder de identificación de tres regiones del código de barras de ADN denominadas rbcL, matK e ITS2.

\section{MATERIALES Y MÉTODOS}

\section{Colecta de muestras en campo}

De acuerdo a datos disponibles de georreferenciación (Villarreal et al. 2008) se establecieron puntos de colecta en la región sureste del estado de Coahuila, México. Se muestrearon un total de 29 árboles. Se colectaron $5 \mathrm{~g}$ de tejido foliar de hojas aparentemente sanas y de brotes jóvenes los cuales se preservaron en alcohol de $96^{\circ}$ para su análisis posterior. Los comprobantes de herbario se prepararon e identificaron morfológicamente siguiendo la guía propuesta por Villarreal et al. (2008) y se depositaron en el Herbario Antonio Narro, Saltillo, México (ANSM). Con base en los resultados de la identificación morfológica, se seleccionaron tres 
ejemplares por especie para el análisis genético.

\section{Extracción, amplificación y secuenciación de ADN}

Se realizaron extracciones de ADN genómico mediante el método modificado de CTAB (Sharma et al. 2003). La concentración y calidad del ADN extraído se comprobó mediante electroforesis en gel de agarosa al $0.8 \%$, a 75 voltios durante $1: 45 \mathrm{~h}$, utilizando un marcador de peso molecular $(1 \mathrm{~Kb}$, Axygen, USA). La visualización del gel se realizó mediante el Sistema de Documentación Gel Axygen (Axygen GD-1000, USA). El ADN se conservó a -20 ${ }^{\circ} \mathrm{C}$ para su análisis posterior. Con el ADN genómico se llevó a cabo la amplificación de los códigos de barras usando los respectivos cebadores (Tabla 1). La reacción en cadena de la polimerasa (PCR) se realizó utilizando la mezcla comercial GoTaq Green Master Mix, 2X ${ }^{T M}$ (Promega, USA), según las especificaciones del fabricante. Las condiciones de la PCR se detallan en la Tabla 1. Los productos de PCR amplificados se verificaron por medio de electroforesis en gel de agarosa $(0.8 \%)$. Los productos de PCR que presentaron bandas inespecíficas, se purificaron utilizando el sistema comercial Wizard SV Gel and PCR Clean Up System ${ }^{T M}$ (Promega, USA), siguiendo las instrucciones del fabricante. Se enviaron $300 \mathrm{ng}$ de producto de PCR de cada muestra al servicio de secuenciación PlateSeq Service (Eurofins ${ }^{T M}$, USA).

\section{Búsqueda, descarga y alineamiento de secuen- cias}

Se descargaron secuencias de especies del género Quercus correspondientes a tres códigos de barras de ADN (rbcL, matK e ITS2) disponibles en la base de datos del BOLSYSTEMS (Boldsystems 2021). Se filtraron secuencias de acuerdo con la longitud de nucleótidos documentada para cada marcador y sin bases ambiguas "N". El alineamiento de las secuencias de referencia y las que se generaron en el laboratorio se llevó a cabo de manera independiente para cada marcador y se ajustaron de forma manual con la herramienta de ClustalW (Thompson et al. 1994) implementado en MEGA X (Tamura et al. 2011).

\section{Análisis de códigos de barras de ADN}

Para evaluar la efectividad del poder discriminatorio de los códigos de barras analizados, se utilizaron los métodos de BLAST, distancia genética y mediante la construcción de árboles de unión de vecinos cercanos, con la finalidad de asignar una identidad a las secuencias analizadas, se determinó la frecuencia con la que cada método devolvió la identidad correcta.

Método de identificación con BLAST. Las secuencias de los tres marcadores se concatenaron (por separado) como una base de datos de referencia y mediante la herramienta de BLASTn (NCBI 2021) se realizó un BLAST considerando un valor de $E<1$ $\times 10^{-5}$ y aciertos máximos de 98 a $100 \%$ con una especie (Ross et al. 2008). Se utilizó el método de distancia (más cercana), con la función BestCloseMatch (Meier et al. 2008) del software SPIDER (Brown et al. 2012).

Con base en las secuencias alienadas, el método de unión del vecino más cercano (Saitou y Nei 1987) en MEGA X (Kumar et al. 2018 y Stecher et al. 2020), se evaluó el desempeño discriminatorio mediante el cálculo de la proporción de especies. Las distancias evolutivas se calcularon utilizando el método de 2 parámetros de Kimura (K2P) (Kimura 1980) y están en las unidades del número de sustituciones de bases por sitio. Todas las posiciones ambiguas se eliminaron para cada par de secuencias (opción de eliminación por pares). Únicamente las especies con múltiples individuos que formaron un clado monofilético en el árbol se consideraron identificadas con éxito. Se construyó un árbol de unión de vecinos para cada marcador. El análisis basado en la construcción de árboles proporciona un método conveniente y visualizado que permite evaluar el desempeño discriminatorio mediante el cálculo de la proporción de grupos monofiléticos.

\section{RESULTADOS}

En cinco localidades de la región sureste del estado de Coahuila, México (Figura 1), se colectaron e identificaron morfológicamente 11 diferentes especies de encinos del subgénero Quercus, de 
Tabla 1. Cebadores y programas de PCR utilizados para la amplificación de ADN.

\begin{tabular}{cccc}
\hline Fragmento & Cebadores (5'-3') & Programa de PCR & Referencia \\
\hline rbcL & rbcLa-F: ATGTCACCACAAACAGAGACTAAAGC & $94^{\circ} \mathrm{C}, 5 \mathrm{~min} ; 35 \times\left(94^{\circ} \mathrm{C}, 30 \mathrm{~s} ;\right.$ & (Kress et al. 2009) \\
& rbcLa-R: GTAAAATCAAGTCCACCRCG & $\left.50^{\circ} \mathrm{C}, 40 \mathrm{~s} ; 72^{\circ} \mathrm{C}, 40 \mathrm{~s}\right) ; 72^{\circ} \mathrm{C}, 10 \mathrm{~min} ; \infty 4^{\circ} \mathrm{C}$. & \\
matK & matK-472f: CCCRTYCATCTGGAATCTTGGTTC & $94^{\circ} \mathrm{C}, 5 \mathrm{~min} ; 35 \times\left(94^{\circ} \mathrm{C}, 15 \mathrm{~s} ; 56^{\circ} \mathrm{C}, 20 \mathrm{~s} ;\right.$ & $($ Yu et al. 2011) \\
& matK-1248r: GCTRTRATAATGAGAAAGATTTCTGC & $\left.72^{\circ} \mathrm{C}, 50 \mathrm{~s}\right) ; 72^{\circ} \mathrm{C}, 10 \mathrm{~min} ; \infty 4^{\circ} \mathrm{C}$. & (Chen et al. 2010) \\
ITS2 & ITS-2F: ATGCGATACTTGGTGTGAAT & $95^{\circ} \mathrm{C}, 4 \mathrm{~min} ; 35 \times\left(94^{\circ} \mathrm{C}, 45 \mathrm{~s} ;\right.$ & \\
& ITS-3R: GACGCTTCTCCAGACTACAAT & $\left.56^{\circ} \mathrm{C}, 1 \mathrm{~min} ; 72^{\circ} \mathrm{C}, 1 \mathrm{~min}\right) ; 72^{\circ} \mathrm{C}, 10 \mathrm{~min} ; \infty 4^{\circ} \mathrm{C}$. & \\
\hline
\end{tabular}

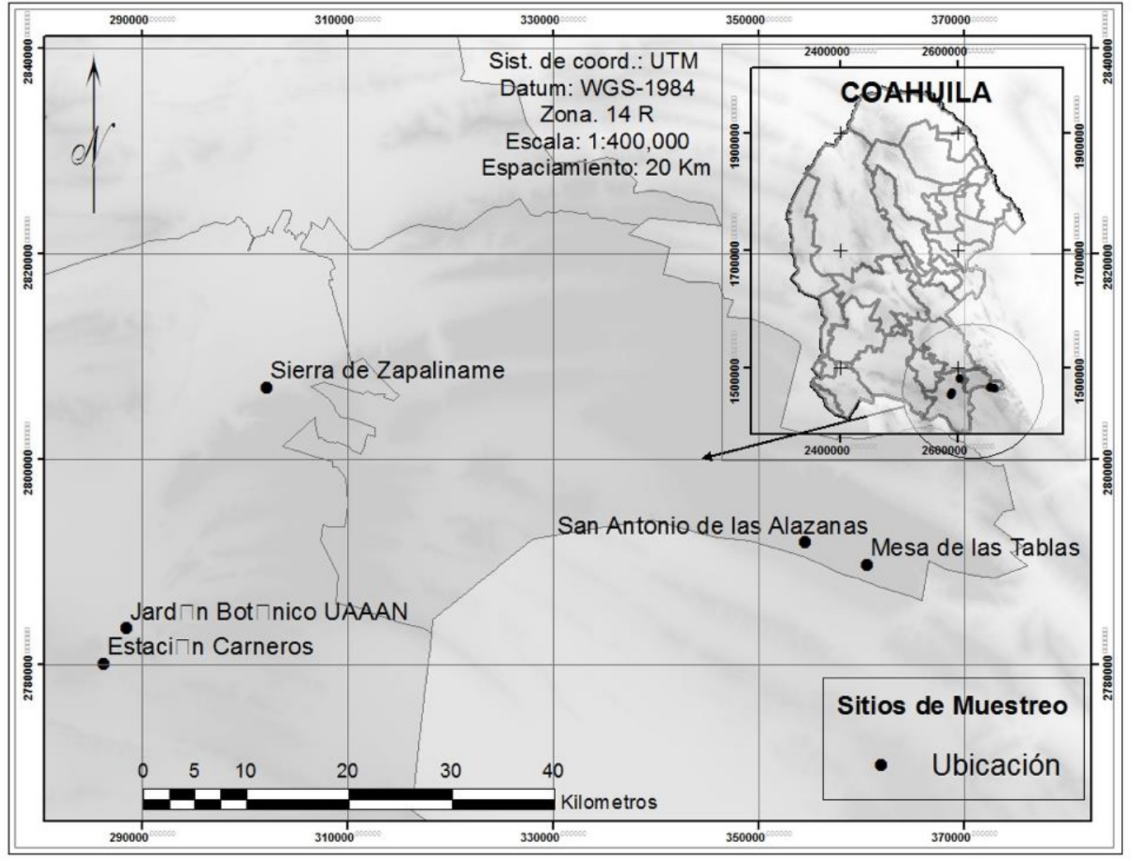

Figura 1. Sitios de recolección de muestras de encinos en la región sureste de Coahuila, México.

las cuales siete pertenecen a la sección Quercus y cuatro a la sección Lobatae (Tabla 2). En total se seleccionaron 29 muestras para los análisis genéticos.

Se obtuvieron productos de PCR en el $100 \%$ de las muestras procesadas, los cuales presentaron el tamaño aproximado esperado en rbcL e ITS2 (600 pb y 400 pb, respectivamente) (Figura 2). En ambos casos la recuperabilidad de las secuencias fue alta ( 96.5 y $86.2 \%$, respectivamente). Los amplicones obtenidos con el fragmento matK resultaron en secuencias de mala calidad que no se incluyeron en el análisis; por lo cual se analizaron únicamente 64 secuencias de referencia matK que cumplieron con los parámetros de calidad.

El análisis incluyó un total de 50 secuencias de consulta 26 del marcador rbcL y 24 del marcador ITS2, provenientes de 11 especies del género Quercus subgénero Quercus, además, se incluyeron 118 especies de referencia (30 rbcL, 27 ITS2 y 61 matK) que provenían de 81 especies del género Quercus (Tabla 3). El conjunto de datos de secuencias del marcador matK incluyó únicamente secuencias de referencia de las regiones de América del Norte y Eurasia. Mientras que los conjuntos de datos rbcL e ITS2 incluyeron secuencias de encinos mexicanos de América del Norte y Eurasia. El análisis involucró diferentes números de secuencias de nucleótidos, por lo que, cada grupo de secuencias tuvo un total diferente de posiciones en el conjunto de datos final de acuerdo con el marcador utilizado. De tal manera que, rbcL generó un total de posiciones de 
Tabla 2. Especies y localización geográfica de muestras colectadas.

\begin{tabular}{|c|c|c|c|c|c|}
\hline Especie & Número de muestra & Latitud N & Longitud O & Altitud & Sección \\
\hline \multirow[t]{3}{*}{ Quercus microphylla } & 1 & $25^{\circ} 15^{\prime} 08.7^{\prime \prime}$ & $100^{\circ} 31^{\prime} 14.39^{\prime \prime}$ & 2388 & Quercus \\
\hline & 2 & $25^{\circ} 15^{\prime} 08.6^{\prime \prime}$ & $100^{\circ} 31^{\prime} 14.6^{\prime \prime}$ & 2391 & Quercus \\
\hline & 3 & $25^{\circ} 15^{\prime} 09.0^{\prime \prime}$ & $100^{\circ} 31^{\prime} 14.4^{\prime \prime}$ & 2391 & Quercus \\
\hline \multirow[t]{3}{*}{ Quecus saltillensis } & 1 & $25^{\circ} 14^{\prime} 13.0^{\prime \prime}$ & $100^{\circ} 26^{\prime} 39.1^{\prime \prime}$ & 2596 & Lobatae \\
\hline & 2 & $25^{\circ} 14^{\prime} 13.5^{\prime \prime}$ & $100^{\circ} 26^{\prime} 39.3^{\prime \prime}$ & 2610 & Lobatae \\
\hline & 3 & $25^{\circ} 14^{\prime} 13.6^{\prime \prime}$ & $100^{\circ} 26^{\prime} 39.2^{\prime \prime}$ & 2608 & Lobatae \\
\hline \multirow[t]{3}{*}{ Quercus hintoniorum } & 1 & $25^{\circ} 13^{\prime} 03.5^{\prime \prime}$ & $100^{\circ} 23^{\prime} 03.1^{\prime \prime}$ & 2864 & Lobatae \\
\hline & 2 & $25^{\circ} 13^{\prime} 04.0^{\prime \prime}$ & $100^{\circ} 23^{\prime} 02.6^{\prime \prime}$ & 2856 & Lobatae \\
\hline & 3 & $25^{\circ} 13^{\prime} 03.8^{\prime \prime}$ & $100^{\circ} 23^{\prime} 02.8^{\prime \prime}$ & 2860 & Lobatae \\
\hline \multirow[t]{3}{*}{ Quercus pringlei } & 1 & $25^{\circ} 07^{\prime} 19.2^{\prime \prime}$ & $101^{\circ} 07^{\prime} 12.1^{\prime \prime}$ & 2315 & Quercus \\
\hline & 2 & $25^{\circ} 07^{\prime} 19.2^{\prime \prime}$ & $101^{\circ} 07^{\prime} 13.2^{\prime \prime}$ & 2316 & Quercus \\
\hline & 3 & $25^{\circ} 07^{\prime} 17.2^{\prime \prime}$ & $101^{\circ} 07^{\prime} 16.4^{\prime \prime}$ & 2333 & Quercus \\
\hline \multirow{3}{*}{ Quercus intricata } & 1 & $25^{\circ} 07^{\prime} 16.0^{\prime \prime}$ & $101^{\circ} 07^{\prime} 16.6^{\prime \prime}$ & 2331 & Quercus \\
\hline & 2 & $25^{\circ} 07^{\prime} 16.5^{\prime \prime}$ & $101^{\circ} 07^{\prime} 16.3^{\prime \prime}$ & 2326 & Quercus \\
\hline & 3 & $25^{\circ} 07^{\prime} 17.0^{\prime \prime}$ & $101^{\circ} 07^{\prime} 17.2^{\prime \prime}$ & 2333 & Quercus \\
\hline \multirow[t]{3}{*}{ Quercus laceyi } & 1 & $25^{\circ} 22^{\prime} 00.5^{\prime \prime}$ & $100^{\circ} 57^{\prime} 58.0^{\prime \prime}$ & 1910 & Quercus \\
\hline & 2 & $25^{\circ} 21^{\prime} 59.8^{\prime \prime}$ & $100^{\circ} 57^{\prime} 58.1^{\prime \prime}$ & 1913 & Quercus \\
\hline & 3 & $25^{\circ} 22^{\prime} 01.8^{\prime \prime}$ & $100^{\circ} 57^{\prime} 56.3^{\prime \prime}$ & 1920 & Quercus \\
\hline \multirow[t]{3}{*}{ Quercus laeta } & 1 & $25^{\circ} 22^{\prime} 02.1^{\prime \prime}$ & $100^{\circ} 57^{\prime} 03.6^{\prime \prime}$ & 1999 & Quercus \\
\hline & 2 & $25^{\circ} 22^{\prime} 01.6^{\prime \prime}$ & $100^{\circ} 57^{\prime} 02.4^{\prime \prime}$ & 2009 & Quercus \\
\hline & 3 & $25^{\circ} 22^{\prime} 01.1^{\prime \prime}$ & $100^{\circ} 57^{\prime} 01.7^{\prime \prime}$ & 2006 & Quercus \\
\hline \multirow[t]{3}{*}{ Quercus laetaxarizonica } & 1 & $25^{\circ} 22^{\prime} 00.4^{\prime \prime}$ & $100^{\circ} 56^{\prime} 54.9^{\prime \prime}$ & 1982 & Quercus \\
\hline & 2 & $25^{\circ} 21^{\prime} 59.9^{\prime \prime}$ & $100^{\circ} 56^{\prime} 54.3^{\prime \prime}$ & 1994 & Quercus \\
\hline & 3 & $25^{\circ} 22^{\prime} 03.7^{\prime \prime}$ & $100^{\circ} 56^{\prime} 50.4^{\prime \prime}$ & 1996 & Quercus \\
\hline \multirow[t]{3}{*}{ Quercus grisea } & 1 & $25^{\circ} 21^{\prime} 59.6^{\prime \prime}$ & $100^{\circ} 56^{\prime} 53.8^{\prime \prime}$ & 2004 & Quercus \\
\hline & 2 & $25^{\circ} 22^{\prime} 01.4^{\prime \prime}$ & $100^{\circ} 57^{\prime} 00.8^{\prime \prime}$ & 1998 & Quercus \\
\hline & 3 & $25^{\circ} 22^{\prime} 04.6^{\prime \prime}$ & $100^{\circ} 56^{\prime} 50.2^{\prime \prime}$ & 2022 & Quercus \\
\hline Quercus hipoxantha & 1 & $25^{\circ} 59^{\prime} 07.0^{\prime \prime}$ & $101^{\circ} 28^{\prime} 41.6^{\prime \prime}$ & 1478 & Lobatae \\
\hline Quercus gravesii & 1 & $25^{\circ} 59^{\prime} 07.3^{\prime \prime}$ & $101^{\circ} 28^{\prime} 41.3^{\prime \prime}$ & 1478 & Lobatae \\
\hline
\end{tabular}
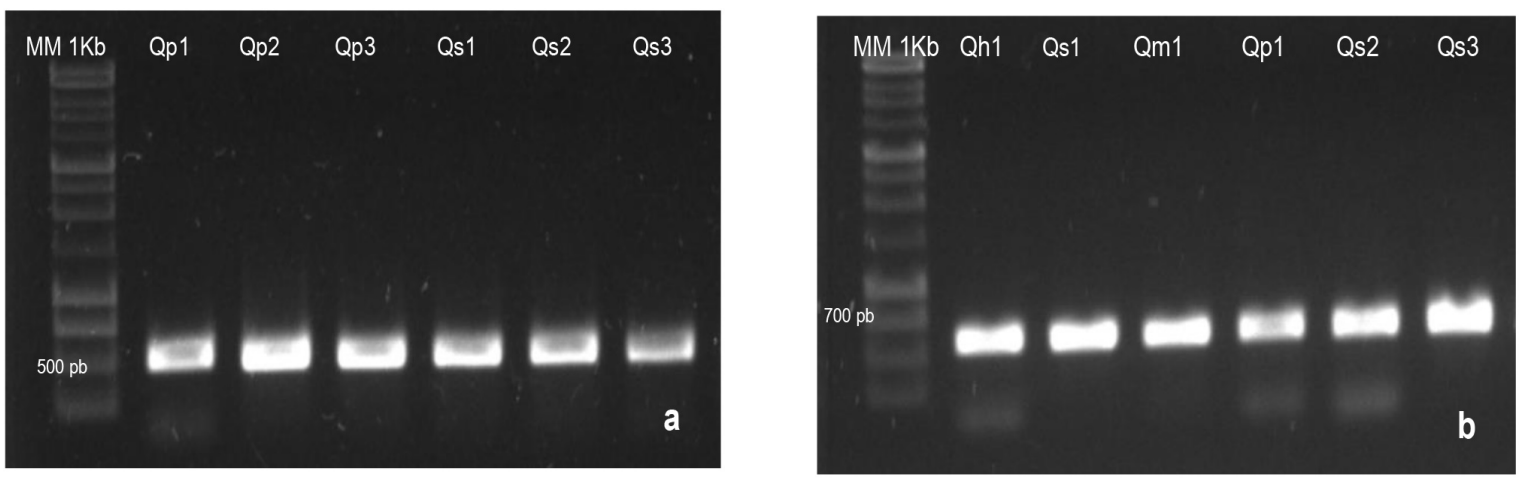

Figura 2. Productos de PCR obtenidos apartir de (a) una región del gen nuclear ITS2 y (b) de una región plastídica del gen rbcL en muestras de ADN de especies de encinos mexicanos.

521 nucleótidos, ITS2 461 nucleótidos y matK 312 nucleótidos.

Para la identificación con el método de BLAST, se construyeron dos bases de datos con las secuencias de consulta, la primera con 26 secuencias rbcL y la otra con las 24 secuencias ITS2, las cuales fueron alineadas de manera independiente junto con las secuencias de referencia. Los resultados indican que, el método de BLAST proporcionó el porcentaje de identificación más alto con promedios de porcentaje de identidad de 98.1 y $98.3 \%$ para los marcadores rbcL e ITS2, respectivamente. Con el método basado en la distancia genética más cercana, los códigos de barras de ADN universal mostraron menos poder discriminatorio, así rbcL, ITS2 y matK tuvieron una tasa de éxito del $3.57,7.84$ y $11.47 \%$, respectivamente. 
Tabla 3. Número de accesión de BoldSystems de secuencias sometidas a comparaciones filogenéticas con tres marcadores.

\begin{tabular}{|c|c|c|c|}
\hline Especie & $\mathrm{rbcL}$ & matK & ITS2 \\
\hline Quercus acutissima & & GBVG072-11 & \\
\hline Quercus afares & & GBVG075-11 & ITSAJ794-14 \\
\hline Quercus alba & GBVG078-11 & GBVG080-11 & \\
\hline Quercus aliena & & GBVG085-11 & \\
\hline Quercus alnifolia & & GBVG091-11 & ITSAJ1237-14 \\
\hline Quercus arizonica & & GBVG094-11 & \\
\hline Quercus aucheri & & GBVG095-11 & ITSAJ1245-14 \\
\hline Quercus baloot & & GBVG096-11 & \\
\hline Quercus baroni & & ITSAH052-14 & \\
\hline Quercus berveridifolia & SDH1252-14 & & \\
\hline Quercus bicolor & MKTRT2706-14 & & \\
\hline Quercus brantii & & GBVG097-11 & ITSAJ803-14 \\
\hline Quercus canariensis & & GBVG099-11 & ITSAJ921-14 \\
\hline Quercus castaneifolia & & GBVG101-11 & ITSAJ806-14 \\
\hline Quercus cedrocencis & SDH1254-14 & & \\
\hline Quercus cerris & GBVG115-11 & GBVG102-11 & ITSAJ812-14 \\
\hline Quercus chrysolepsis & & GBVS274-13 & \\
\hline Quercus coccifera & GBVG135-11 & GBVG121-11 & ITSAJ1261-14 \\
\hline Quercus cornelius muelleri & SDH3391-15 & & \\
\hline Quercus corrugata & MHPAC1141-11 & & \\
\hline Quercus crenata & GBVG149-11 & GBVG140-11 & \\
\hline Quercus dentata & & GBVG151-11 & \\
\hline Quercus ellipsodalis & HIMS059-12 & & \\
\hline Quercus engelmanii & SDH3367-15 & & \\
\hline Quercus fabri & & GBVG157-11 & \\
\hline Quercus faginea & & GBVG158-11 & ITSAJ959-14 \\
\hline Quercus floribunda & & GBVG160-11 & ITSAJ1295-14 \\
\hline Quercus frainetto & & GBVG161-11 & ITSAJ977-14 \\
\hline Quercus gambelli & & GBVG174-11 & \\
\hline Quercus garryana & VPSBC1009-13 & GBVG176-11 & \\
\hline Quercus glauca & GBVG185-11 & GBVS270-13 & \\
\hline Quercus griffithii & & GBVG187-11 & \\
\hline Quercus ilex & ATG011-14 & GBVG194-11 & ITSAJ1306-14 \\
\hline Quercus ilicifolia & VASCA016-15 & & \\
\hline Quercus infectoria & API328-14 & GBVG212-11 & \\
\hline Quercus infectoria var. boissieri & & & ITSAJ912-14 \\
\hline Quercus infectoria var. infectoria & & & ITSAJ1034-14 \\
\hline Quercus ithaburensis var. ithaburensis & & GBVG218-11 & \\
\hline Quercus ithaburensis var. macrolepsis & & GBVG219-11 & ITSAJ845-14 \\
\hline Quercus kellogi & SDH1261-14 & & \\
\hline Quercus laeta & & GBVG222-11 & \\
\hline Quercus lanata var. lanata & & GBVW3164-13 & \\
\hline Quercus leucotrichophora & & GBVW3167-13 & \\
\hline Quercus libani & & GBVG223-11 & \\
\hline Quercus lobata & & GBVG225-11 & \\
\hline Quercus lusinatica & & GBVG226-11 & \\
\hline Quercus macranthera & & GBVG229-11 & ITSAJ1055-14 \\
\hline Quercus macrocarpa & FOND165-12 & GBVG233-11 & \\
\hline Quercus mongolica & RINGV016-12 & GBVG237-11 & \\
\hline Quercus montana & & GBVG262-11 & \\
\hline Quercus muhelenbergi & MKTRT534-12 & & \\
\hline Quercus petraea & GBVG295-11 & GBVG278-11 & \\
\hline Quercus petraea var. iberica & & GBVG299-11 & \\
\hline Quercus petrea var. petrea & & GBVS1218-13 & ITSAJ1013-14 \\
\hline Quercus phillyraeoides & & GBVG302-11 & \\
\hline Quercus polycarpa & & GBVG307-11 & \\
\hline Quercus pontica & & GBVG308-11 & ITSAJ1121-14 \\
\hline Quercus prinoides & MKTRT558-12 & & \\
\hline
\end{tabular}




\begin{tabular}{|c|c|c|c|}
\hline Quercus pseudosemercarpifolia & & GBVG309-11 & \\
\hline Quercus pubescens & GBVG326-11 & GBVG310-11 & ITSAJ1131-14 \\
\hline Quercus pubescens var. pubescens & & GBVS1219-13 & \\
\hline Quercus pyrenaica & & GBVG329-11 & ITSAJ1168-14 \\
\hline Quercus robur & GBVG349-11 & GBVG330-11 & \\
\hline Quercus robur var. robur & & GBVG354-11 & \\
\hline Quercus robur var. pedunculiflora & & & ITSAJ1074-14 \\
\hline Quercus rubra & BPNP195-08 & GBVS271-13 & \\
\hline Quercus semecarpifolia & & GBVG372-11 & \\
\hline Quercus serrata & & GBVG373-11 & \\
\hline Quercus serrata var. brevipetiolata & GBVG385-11 & GBVG384-11 & \\
\hline Quercus shumardii & MKTRT582-12 & & \\
\hline Quercus stewardiana & & GBVX5226-15 & \\
\hline Quercus suber & GBVG404-11 & GBVG390-11 & ITSAJ852-14 \\
\hline Quercus suber x Quercus trojana & & ITSAJ891-14 & \\
\hline Quercus trojana & & GBVG408-11 & ITSAJ896-14 \\
\hline Quercus variabilis & & GBVG415-11 & \\
\hline Quercus vulcanica & & GBVG419-11 & ITSAJ1226-14 \\
\hline Quercus wislizeni var. frutescens & SDH3355-15 & & \\
\hline Quercus wislizeni var. wislizeni & SDH1265-14 & & \\
\hline Quercus wutaishanica & & GBVG420-11 & \\
\hline Quercus $x$ ganderi & SDH3343-15 & & \\
\hline Quercus $x$ morisii & & GBVG422-11 & \\
\hline
\end{tabular}

Con base en el método de unión de vecinos cercanos, los taxones de plantas recolectados se agruparon de acuerdo con el análisis de similitud, en cada conjunto de datos se formaron diferentes grupos monofiléticos. En general, en los árboles se aprecian grupos monofiléticos definidos. El porcentaje de discriminación más bajo se generó con el marcador rbcL (64.28\%) (Figura 3). Con el marcador ITS2 se logró un porcentaje de discriminación del $80.39 \%$, en la Figura 4 se aprecian algunos grupos monofiléticos. Mientras que, con el marcador matK se obtuvo el porcentaje más alto (81.96\%), en la Figura 5 se confirma la separación de las 61 especies por secciones del subgénero y por regiones geográficas, formando tres clados pincipales correspondientes a las secciones Quercus (Eurasia), Cerris (Eurasia) y Quercus (América del Norte). Teniendo en cuenta que en los conjuntos de datos de los marcadores rbcL e ITS2 contenían secuencias de especies mexicanas, ITS2 proporcionó una mejor resolución cladística en este grupo de plantas. Cada árbol generado mostró formas diferentes de acuerdo al agrupamiento de especies estrechamente relacionadas, mientras que las especies lejanamente relacionadas se dispersaron relativamente.

\section{DISCUSIÓN}

Las características que un código de barras ideal debería poseer son: cebadores altamente universales y de secuencia corta, pero con suficiente variación entre secuencias que permita la identificación y discriminación entre especies (Erickson et al. 2008, Kress y Erickson 2008, Hollingsworth et al. 2009, Stoeckle et al. 2011). El Grupo de Trabajo CBOL propuso dos regiones del cloroplasto (rbcL y matK), como los códigos de barras centrales, esto, debido a la conservación de sus genes y a la universalidad de amplificación de los cebadores utilizados (De Groot et al. 2011, Pang et al. 2012 y Saarela et al. 2013). Los códigos de barras seleccionados en este estudio presentaron resultados variables. La secuencia rbcL mostró una cobertura alta de amplificación (100\%) y secuenciación (96.5\%) en las once especies de encinos mexicanos, resultados similares han sido reportados en diferentes grupos de plantas de especies arbóreas, tropicales y medicinales (Gonzalez et al. 2009, Parmentier et al. 2013, Kang et al. 2017, Maloukh et al. 2017). De igual manera en encinos italianos, se obtuvieron resultados positivos en la amplificación del ADN (Piredda et al. 2011). Lo anterior, confirma que es posible la amplificación y 
Pacheco-Reyes et al.

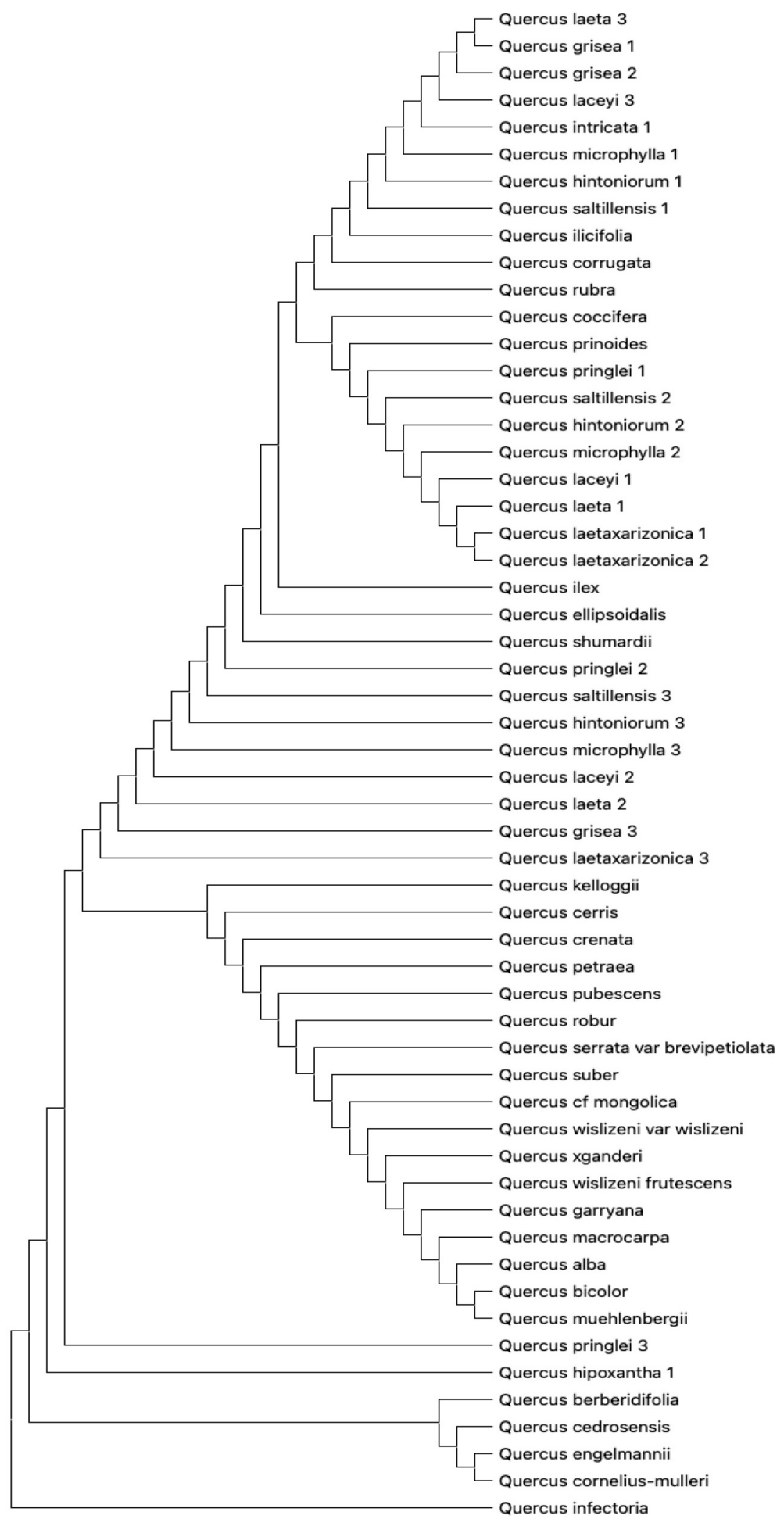

Figura 3. Análisis filogenético molecular usando la región plastídica rbcL para 56 secuencias de nucleótidos con base en la distancia genética K2P. 


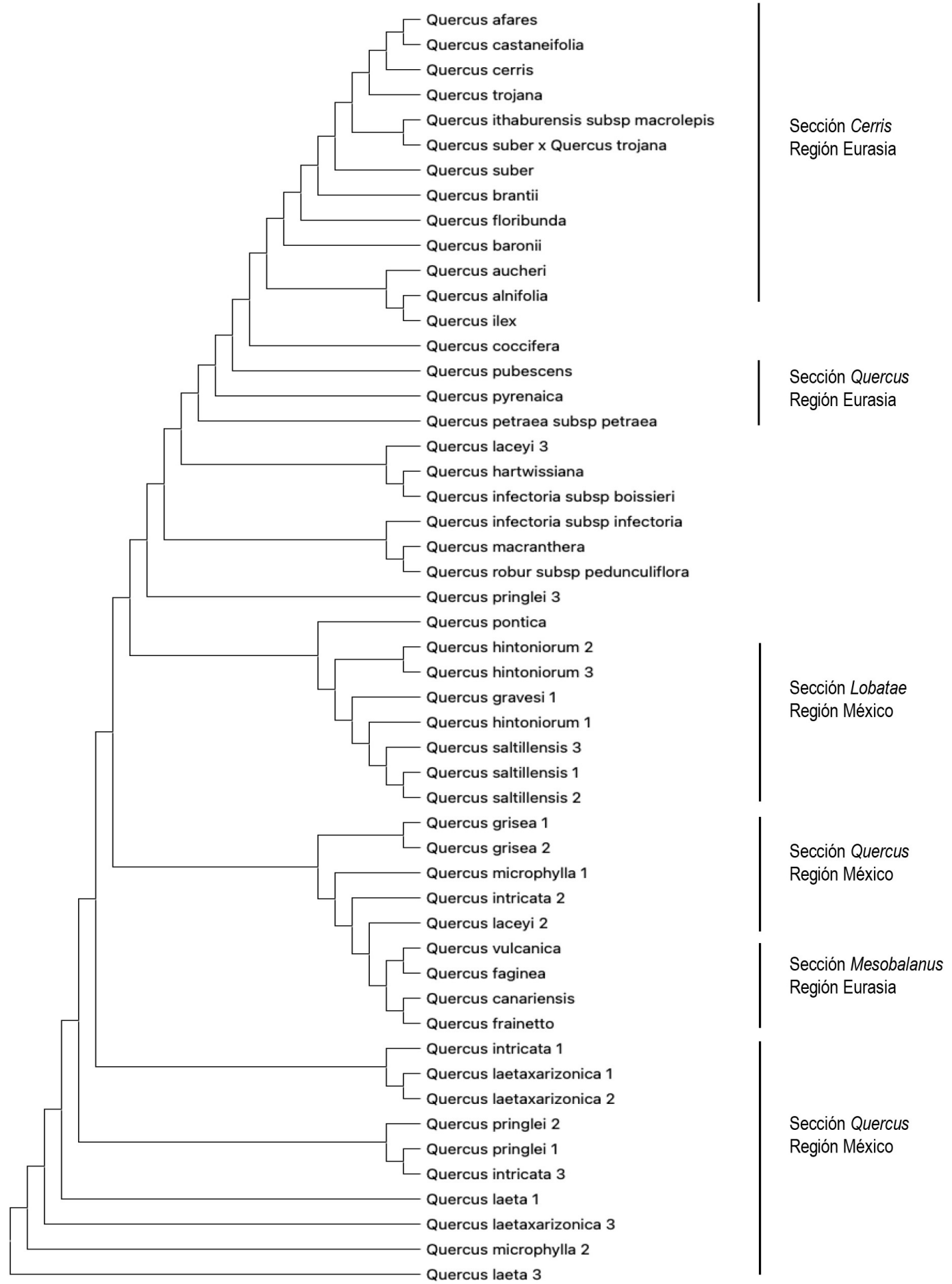

Figura 4. Análisis filogenético molecuar usando la región nuclear ITS2 para 51 secuencias de nucleótidos con base en la distancia genética K2P. 
Pacheco-Reyes et al. PCOSIstemas

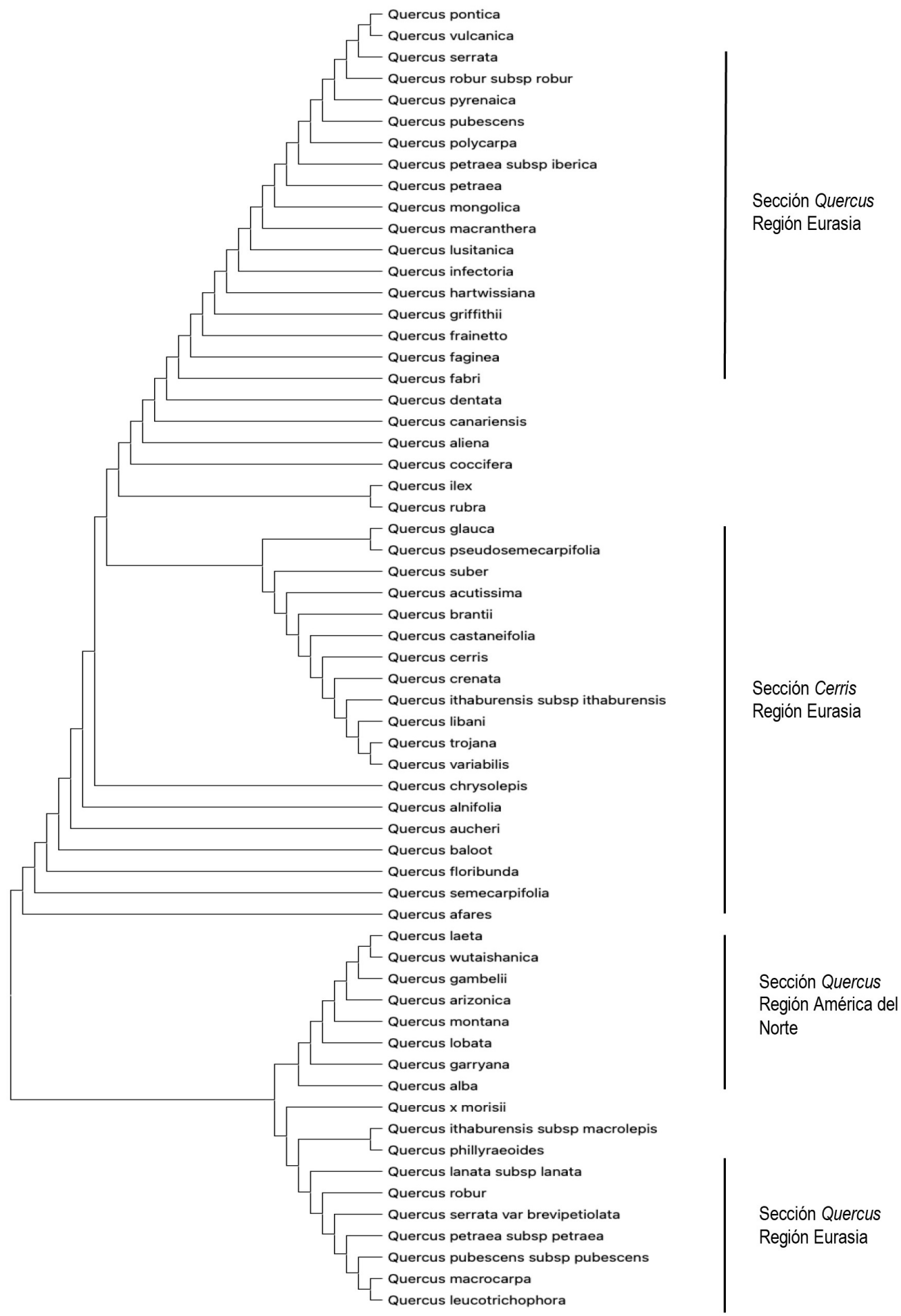

Figura 5. Análisis filogenético molecular usando la región plastídica matK para 61 secuencias de nucleótidos con base en la distancia genética K2P. 
secuenciación de buena calidad, en condiciones estándar y en una amplia gama de taxones con cebadores rbcL utilizados en este estudio. Por otro lado, el bajo éxito de amplificación y secuenciación utilizando cebadores matK se ha informado en diferentes grupos de plantas, en angiospermas y gimnospermas, incluso en grupos de plantas específicos (Kress y Erickson 2008, Bafeel et al. 2011, Maloukh et al. 2017, Amandita et al. 2019). Mientras que Piredda et al. (2011), al eliminar de sus análisis los datos de secuencias provenientes de la región matK (universal) sugerida por el $\mathrm{CBOL}$ en encinos italianos, debido a la baja amplificación por PCR, incluso con cebadores específicos para dicho taxón no obtuvieron buenos resultados de amplificación y su poder discriminatorio fue relativamente bajo. Mientras que Yang et al. (2016) reportaron una alta tasa de amplificación con cebadores diseñados para el grupo de plantas de Quercus de la región de China, pero con bajo rendimiento en la identificación de especies. Resultados alentadores se han logrado en algunos grupos de plantas específicos como Asteraceae (Gao et al. 2010), Lamiaceae (De Mattia et al. 2011), Tetrastigma (Fu et al. 2011) y en palmas (Jeanson et al. 2011). La variabilidad de resultados obtenidos con esta región, puede deberse a la diversa tasa evolutiva en diferentes taxones, ya que la región matK tiene una alta tasa de sustituciones de nucleótidos que puede estar influenciada por factores climáticos que afecta la conservación del locus de diferentes grupos de plantas (Hilu et al. 2003, Gillman et al. 2010). La utilidad de la región matK como código de barras de ADN es fundamental en la discriminación e identificación de especies, por lo que, es imprescindible continuar con la búsqueda de cebadores que permitan la amplificación de ADN de grupos taxonómicos difíciles o grupos de plantas específicos (Hollingsworth et al. 2011, Piredda et al. 2011). En 2011 el CBOL propuso la región ITS como un código de barras potencialmente útil para la identificación de especies de plantas (China Plant BOL Group et al. 2011). Su utilidad ha sido respaldada en diferentes investigaciones que incluyen una amplia gama de grupos taxonómicos donde se han logrado amplificaciones exitosas y secuencias de alta calidad
(Bellarosa et al. 2005, Chen et al. 2010, Pang et al. 2010, Simeone et al. 2013, Chen et al. 2015). Aúnque los resultados de amplificación y secuenciación pueden ser variables según el taxón de plantas (Kang et al. 2017). En este estudio los cebadores ITS2 presentaron una tasa de amplificación del 100\% y permitieron obtener secuencias de alta calidad en un $86.2 \%$ de las muestras analizadas. La característica principal de la región ITS2 es que es una secuencia corta, lo cual permite una alta tasa de amplificación, además, sus estructuras secundarias se conservan y proporcionan información útil en biología (Meyer y Paulay 2005 y Chen et al. 2010). Sin embargo, a pesar del desarrollo de la tecnología de los códigos de barras de ADN, aún no se cuenta con un código estándar para la identificación de taxones de plantas (Ballardini et al. 2013, Hollingsworth et al. 2011).

Uno de los propósitos principales de la tecnología de códigos de barras de ADN es la identificación de especies desconocidas, de tal manera que, una secuencia de código de barras particular coincida con secuencias confirmadas disponibles en las bases de datos. Al respecto los tres métodos de análisis utilizados proporcionaron diferentes tasas de discriminación en las especies de encinos del subgénero Quercus. Teniendo en cuenta que, los conjuntos de datos de los marcadores rbcL e ITS2 contenían secuencias de consulta de especies mexicanas, el número de las coincidencias correctas en BLAST proporcionó el poder de identificación alto con 98.1 y $98.3 \%$, respectivamente. De igual manera, en un grupo de encinos de origen chino y en un grupo taxonómico específico (Apocynaceae) se encontró que el método de BLAST mostró la identificación más alta, respecto a otros métodos de identificación a nivel de género y especie (Cabellin et al. 2016, Yang et al. 2016).

El método basado en la distancia genética mostró tasas de discriminación extremadamente bajas para las especies de encinos $3.57 \%$ con rbcL, $7.84 \%$ con ITS2 y $11.47 \%$ con matK. Resultados similares obtuvieron Pang et al. (2019) en donde, la distancia genética mostró las tasas de discriminación más bajas para las especies de encinos italianos con 
porcentajes que van del $12.50 \%$ para rbcL y $25 \%$ para el marcador matK. Por otro lado, en especies de encino chino el método basado en la distancia genética también mostró tasas de discriminación bajas $(0$ al 17.14\%) (Pang et al. 2010). En contraste, Chen et al. (2010) obtuvieron resultados favorables cuando utilizaron el método de distancia genética más cercana, aplicado a especies de plantas medicinales con el marcador ITS2, el cual identificó correctamente el 90.3 y el $99.7 \%$ de las muestras a nivel de especie y género, respectivamente. En este estudio la aplicación de este método parece poco práctico en la codificación de especies de encinos dado que las distancias genéticas intra e interespecíficas se superponen en gran medida. El análisis basado en la distancia genética ha sido propuesto como un método altamente sensible a procesos de divergencia reciente entre especies, lo que puede resultar en una clasificación de linajes incompletos, y afectar el resultado obtenido aplicando dicho método (Simeone et al. 2013). En el caso específico de encinos diferentes autores mencionan la baja identificación basada en la distancia genética, debido a que, un muestreo mayor puede conducir a distancias genéticas superpuestas, además de la frecuente hibridación entre especies y una baja tasa de variación (Piredda et al. 2011, Simeone et al. 2013).

Junto con los datos moleculares de los códigos de barras de ADN, la construcción de árboles se utilizó para probar las relaciones filogenéticas de las cinco secciones del subgénero Quercus basadas en la morfología, además de probar la separación de grupos por regiones geográficas. Mediante la metodología de unión de vecinos cercanos y distancias evolutivas para 92 especies de encinos se generaron clados individuales de acuerdo al marcador utilizado. Los resultados de los árboles proporcionaron información importante sobre las relaciones filogenéticas de especies de encinos. Este método mostró tasas de discriminación relativamente altas. Los encinos (Quercus L.) pertenecen a un grupo de plantas con alta complejidad taxonómica, actualmente reconocidos dos subgéneros: Quercus distribuido principalmente en América del Norte y Eurasia (región del Mediterrá- neo) y el subgénero Cyclobalanopsis distribuido principalmente en el sudeste asiático. En este estudio, los árboles filogenéticos confirmaron la separación de grupos monofiléticos de acuerdo con datos morfológicos (Valencia 2004, Oh y Manos 2008), en secciones del grupo Quercus (Cerris, Lobatae, Mesobalanus, Protobalanus y Quercus) con el uso de los marcadores ITS2 y matK los cuales generaron un poder de discriminación del 80.39 y $81.96 \%$, respectivamente (Figuras 4 y 5). Resultados similares se han obtenido en diferentes grupos de plantas (Amandita et al. 2019, Chen et al. 2010). La variación en el poder de identificación se debe probablemente a la diferenciación en las tasas de mutación y evolución que resulta en una menor información genética de los marcadores (Palmer et al. 2000, Yang et al. 2017). Con lo anterior, se confirma que la inclusión de la región ITS2 como código de barras de ADN parece ser esencial en la discriminación de grupos de plantas estrechamente relacionados (Chen et al. 2010, Simeone et al. 2013). Además, en el árbol generado con secuencias del marcador matK se observa una separación de especies de acuerdo a su centro de diversificación, americano o euroasiático (Figura 5), lo anterior, respalda la posibilidad que las especies de encinos provienen de un doble origen (América y Eurasia) (Oh y Manos 2008, Hubert et al. 2014, Yang et al. 2017). Por otro lado, el marcador rbcL indicó limitaciones en su poder de identificación, con un porcentaje bajo (64.28\%), lo cual condujo a un árbol poco claro en la generación de grupos monofiléticos (Figura 3). Este resultado puede deberse al hecho de que, a pesar de que la región rbcL tiene una amplificación casi universal, su tasa de evolución es relativamente baja (Simeone et al. 2013).

México destaca como un país con una diversificación excepcional de encinos asociado a una mayor heterogeneidad ecológica. La disponibilidad de humedad en los nichos ecológicos, afecta directamente las tasas de evolución y diversificación de linajes de encinos en México, en comparación con la región de América del Norte (Torres et al. 2011 y Hipp et al. 2018). Finalmente, la comparación de marcadores rbcL e ITS2 respecto a secuencias de encinos de origen mexicano permite inferir que el 
marcador ITS2 proporciona una mejor identificación y agrupamiento de estas especies de acuerdo con datos morfológicos (Valencia 2004, Villarreal et al. 2008).

\section{CONCLUSIONES}

Con base en los resultados obtenidos con la metodología de unión de vecinos cercanos, altos porcentajes de amplificación y recuperabilidad de secuencias, es concluyente que el marcador de la región nuclear ITS2 funciona razonablemente bien para la identificación especies de encinos. De igual manera la región matK tuvo un buen desempeño de acuerdo a la discriminación de especies obtenida, pero su universalidad de amplificación en un amplio grupo de plantas aún es cuestionable. Mientras que el marcador rbcL es ampliamente utilizado por sus características de amplificación y obtención de secuencias, su baja tasa evolutiva no permite un uso eficiente de dicho marcador en la identificación de especies. Es importante mencionar que, según datos del BOLSYSTEMS, únicamente alrededor del $5 \%$ de especies de encinos mexicanos cuentan con al menos un código de barras de ADN, lo que muestra la importancia de continuar y expandir los estudios de esta índole para las especies de encinos de México.

\section{AGRADECIMIENTOS}

Al Consejo Nacional de Ciencia y Tecnología (CONACYT) por su apoyo a través de una beca de doctorado. A la Secretaria de Investigación y Posgrado del IPN por la financiación parcial a través del proyecto 20201683. Al Dr. Juan Antonio Encina Domínguez por su contribución en la identificación morfológica de las especies de encinos analizadas. Al ingeniero Gustavo Alejandro De la Torre Mendoza por su apoyo en la realización del mapa de sitios de colecta.

\section{LITERATURA CITADA}

Amandita FY, Rembold K, Vornam B, Rahayu S, Siregar IZ, Kreft H, et al. (2019) DNA barcoding of flowering plants in Sumatra, Indonesia. Ecology and Evolution 9: 1858-1868.

Bafeel SO, Arif IA, Bakir MA, Khan HA, Al Farhan AH, Al Homaidan AA, Ahamed A, Thomas J (2011) Comparative evaluation of PCR success with universal primers of maturase $\mathrm{K}$ (matK) and ribulose-1, 5-bisphosphate carboxylase oxygenase large subunit (rbcL) for barcoding of some arid plants. Plant Omics. Research Note 4: 195-198.

Ballardini M, Mercuri A, Littardi C, Abbas S, Couderc M, Ludeña B, Jean-Christophe P (2013) The chloroplast DNA locus psbZ-trnfM as a potential barcode marker in Phoenix L. (Arecaceae). ZooKeys 365: 71-82.

Bellarosa R, Simeone MC, Papini A, Schirone B (2005) Utility of ITS sequence data for phylogenetic reconstruction of Italian Quercus spp. Molecular Phylogenetics and Evolution 34: 355-370.

Borek K, Summer S (2009) DNA barcoding of Quercus sp. at Pierce Cedar Creek Institue using the matK gene. In: Grand Rapids: Aquinas College. Biology Deparment. Michigan, USA. https://www.cedarcreekinstitute.org/ PDF/Botany/2009\%20-\%20DNA\%20barcoding\%20of\%20Quercus\%20\%20sp.\%20at\%20Pierce\%20Cedar\% 20Creek\%20Institute\%20using\%20the\%20matK\%20gene.pdf. Fecha de consulta: 25 de enero de 2021.

Brown S, Collins R, Boyer S, Lefort M, Malumbres-Olarte J, Vink C, Cruickshank R (2012) Spider: An R package for the analysis of species identity and evolution, with particular reference to DNA barcoding. Molecular Ecology Resources 12: 562-565.

Boldsystems (2021) Barcode of life data systems. https://www.boldsystems.org/. Fecha de consulta: 20 de diciembre de 2020. 
Cabelin V, Alejandro G (2016) Efficiency of matK, rbcL, trnH-psbA, and trnL-F (cpDNA) to molecularly authenticate Philippine ethnomedicinal Apocynaceae through DNA barcoding. Pharmacognosy magazine, 12(Suppl 3): S384. DOI: 10.4103/0973-1296.185780.

Chen J, Zhao J, Erickson DL, Xia N, Kress WJ (2015) Testing DNA barcodes in closely related species of Curcuma (Zingiberaceae) from Myanmar and China. Molecular Ecology Resources 15: 337-348.

Chen S, Yao H, Han J, Liu C, Song J, Shi L, Zhu Y, Ma X, Gao T, Pang X, Luo K, Li Y, Li X, Jia X, Lin Y, Leon C (2010) Validation of the ITS2 region as a novel DNA barcode for identifying medicinal plant species. PloS One 5: 8613. DOI: 10.1371/journal.pone.0008613.

China Plant BOL, Li DZ, Gao LM, Li HT, Wang H, Ge XJ, Liu JQ, Chen ZD, Yang JB, Fu CX, Zeng CX, Yan HF, Zhu YJ, Sun YS, Chen SY, Zhao L, Wang K, Yang T, Duan GW (2011) Comparative analysis of a large dataset indicates that internal transcribed spacer (ITS) should be incorporated into the core barcode for seed plants. Proceedings of the National Academy of Sciences of the United States of America 108: 19641-19646.

Costion C, Ford A, Cross H, Crayn D, Harrington M, Lowe A (2011) Plant DNA barcodes can accurately estimate species richness in poorly known floras. PloS One 6: e26841. DOI: 10.1371/journal.pone.0026841

De Groot GA, During HJ, Maas JW, Schneider H, Vogel JC, Erkens RH (2011) Use of rbcL and trnL-F as a two-locus DNA barcode for identification of NW-European ferns: an ecological perspective. PLoS One 6: e16371. DOI: 10.1371/journal.pone.0016371.

De Mattia F, Bruni I, Galimberti A, Cattaneo F, Casiraghi M, Labra M (2011) A comparative study of different DNA barcoding markers for the identification of some members of Lamiacaea. Food Research International 44: 693-702.

Erickson DL, Spouge J, Resch A, Weigt LA, Kress JW (2008) DNA barcoding in land plants: developing standards to quantify and maximize success. Taxon 57: 1304-1316.

Fu Y, Jiang W, Fu C (2011) Identification of species within Tetrastigma (Miq.) Planch.(Vitaceae) based on DNA barcoding techniques. Journal of Systematics and Evolution 49: 237-245.

Gao T, Yao H, Song J, Liu C, Zhu Y, Ma X, Pang X, Xu H, Chen S (2010) Identification of medicinal plants in the family Fabaceae using a potential DNA barcode ITS2. Journal of Ethnopharmacology 130: 116-121.

Gillman LN, Keeling DJ, Gardner RC, Wright SD (2010) Faster evolution of highly conserved DNA in tropical plants. Journal of Evolutionary Biology 23: 1327-1330.

Gonzalez MA, Baraloto C, Engel J, Mori SA, Pétronelli P, Riéra B, Roger A, Thébaud C, Chave J (2009) Identification of Amazonian trees with DNA barcodes. PLoS One 4: e7483. DOI: 10.1371/journal.pone.0007483.

Govaerts R, Frodin DG (1998) World Checklist and Bibliography of Fagales (Betulaceae, Corylaceae, Fagaceae and Ticodendraceae). Royal Botanic Gardens, Kew. Vol. 2. United Kingdom. 407p.

Govindaraghavan S, Hennell JR, Sucher NJ (2012) From classical taxonomy to genome and metabolome: towards comprehensive quality standards for medicinal herb raw materials and extracts. Fitoterapia 83: 979988.

CBOL Plant Working Group, Hollingsworth PM, Forrest LL, Spouge JL, Hajibabaei M, Ratnasingham S, Van der Bank M, Chase MW, Cowan RS, Erickson DL, et al. (2009) A DNA barcode for land plants. Proceedings of the National Academy of Sciences 106: 12794-12797.

Gual-Díaz M (2014) La familia Fagaceae en el bosque mesófilo de montaña de México. Botanical Sciences 92 : 193-204. 
Hajibabaei M, Singer GAC, Hebert PDN, Hickey DA (2007) DNA barcoding: how it complements taxonomy, molecular phylogenetics and population genetics. TRENDS in Genetics 23: 167-172.

Hebert PDN, Cywinska A, Ball SL, deWaard JR (2003) Biological identifications through DNA barcodes. Proceedings. Biological Sciences 270: 313-321.

Hilu KW, Borsch T, Müller K, Soltis DE, Soltis PS, Savolainen V, Chase MW, Powell MP, Alice LA, Evans R, Sauquet H, Neinhuis C, Slotta TAB, Rohwer JG, Campbell CS, Chatrou LW (2003) Angiosperm phylogeny based on $<011>$ matK sequence information. American Journal of Botany 90: 1758-1776.

Hipp AL, Manos PS, Cavender-Bares J, Eaton DA, Nipper R (2013) Using phylogenomics to infer the evolutionary history of oaks. International Oak Journal 24: 61-71.

Hipp AL, Manos PS, González-Rodríguez A, Hahn M, Kaproth M, McVay JD, Avalos SV, Bares-Cavender J (2018) Sympatric parallel diversification of major oak clades in the Americas and the origins of Mexican species diversity. New Phytologist 217: 439-452.

Hoban SM, McCleary TS, Schlarbaum SE, Romero-Severson J (2009) Geographically extensive hybridization between the forest trees American butternut and Japanese walnut. Biology Letters 5: 324-327.

Hollingsworth ML, Andra A, Forrest LL, Richardson J, Pennington RT, Long DG, Cowan R, Chase MW, Gaudeul M, Hollingsworth PM (2009) Selecting barcoding loci for plants: evaluation of seven candidate loci with species-level sampling in three divergent groups of land plants. Molecular Ecology Resources 9: 439-457.

Hollingsworth PM, Graham SW, Little DP (2011) Choosing and using a plant DNA barcode. PloS One 6: e19254. DOI: $10.1371 /$ journal.pone.0019254.

Hubert F, Grimm GW, Jousselin E, Berry V, Franc A, Kremer A (2014) Multiple nuclear genes stabilize the phylogenetic backbone of the genus Quercus. Systematics and Biodiversity 12: 405-423.

Jeanson ML, Labat JN, Little DP (2011) DNA barcoding: a new tool for palm taxonomists? Annals of Botany 108 : 1445-1451.

Kang Y, Deng Z, Zang R, Long W (2017) DNA barcoding analysis and phylogenetic relationships of tree species in tropical cloud forests. Scientific Reports 7: 12564. DOI: 10.1038/s41598-017-13057-0.

Kimura M (1980) A simple method for estimating evolutionary rates of base substitutions through comparative studies of nucleotide sequences. Journal of Molecular Evolution 16: 111-120.

Kress WJ, Erickson DL (2008) DNA barcodes: genes, genomics, and bioinformatics. Proceedings of the National Academy of Sciences 105: 2761-2762.

Kress WJ, Erickson DL, Jones FA, Swenson NG, Perez R, Sanjur O, Bermingham E (2009) Plant DNA barcodes and a community phylogeny of a tropical forest dynamics plot in Panama. Proceedings of the National Academy of Sciences 106: 18621-18626.

Kumar S, Stecher G, Li M, Knyaz, Tamura K (2018) MEGA X: molecular evolutionary genetics analysis across computing platforms. Molecular Biology and Evolution 35: 1547-1549.

Maloukh L, Kumarappan A, Jarrar M, Salehi J, El-Wakil H, Lakshmi TVR (2017) Discriminatory power of rbcL barcode locus for authentication of some of United Arab Emirates (UAE) native plants. 3 Biotech 7: 144. DOI: 10.1007 / s13205-017-0746-1.

Manos PS, Doyle JJ, Nixon KC (1999) Phylogeny, biogeography, and processes of molecular differentiation in Quercus subgenus Quercus (Fagaceae). Molecular Phylogenetics and Evolution 12: 333-349. 
Meier R, Zhang G, Ali F (2008) The use of mean instead of smallest interspecific distances exaggerates the size of the "barcoding gap" and leads to misidentification. Systematic biology 57: 809-813.

Meyer CP, Paulay G (2005) DNA barcoding: error rates based on comprehensive sampling. PLoS Biol 3: e422. DOI: https://doi.org/10.1371/journal.pbio.0030422.

NCBI (2021) National Center for Biotechnology Information. https://blast.ncbi.nlm.nih.gov/Blast.cgi?PROGRAM= blastn\&PAGE_TYPE=BlastSearch\&LINK_LOC=blasthome. Fecha de consulta: 10 de diciembre de 2020.

Oh SH, Manos PS (2008) Molecular phylogenetics and cupule evolution in Fagaceae as inferred from nuclear CRABS CLAW sequences. Taxon 57: 434-451.

Palmer JD, Adams KL, Cho Y, Parkinson CL, Quu YL, Song K (2000) Dynamic evolution of plant mitochondrial genomes: mobile genes and introns and highly variable mutation rates. Proceedings of the National Academy of Sciences 97: 6960-6966.

Pang X, Liu C, Shi L, Li R, Liang D, Li H, Cherny SS, Chen S (2012) Utility of the trnH?psbA intergenic spacer region and its combinations as plant DNA barcodes: a meta-analysis. PloS One 7: e48833. DOI: 10.1371/journal.pone.0048833.

Pang X, Song J, Zhu Y, Xie C, Chen S (2010) Using DNA barcoding to identify species within Euphorbiaceae. Planta Medica 76: 1784-1786.

Parmentier I, Duminil J, Kuzmina M, Philippe M, Thomas DW, Kenfack D, Chuyong GB, Cruaud C, Hardy OJ (2013) How effective are DNA barcodes in the identification of African rainforest trees?. PLoS One 8: e54921. DOI: 10.1371 / journal.pone.0054921.

Pérez Mojica E (2017) Estudio preliminar del género Quercus (Fagaceae) en Tamaulipas, México. Acta Botánica Mexicana 120: 59-111.

Piredda R, Simeone MC, Attimonelli M, Bellarosa R, Schirone B (2011) Prospects of barcoding the Italian wild dendroflora: oaks reveal severe limitations to tracking species identity. Molecular Ecology Resources 11: $72 ? 83$.

Ramamoorthy TP, Bye R, Lot A, Fa J, Conzalez-Montagut R (1993) Biological diversity of Mexico: origins and distribution. Oxford University Press New York. EEUU. 812p.

Romero-Rangel S (2006) Revisión taxonómica del complejo Acutifoliae de Quercus (Fagaceae) con énfasis en su representación en México. Acta Botánica Mexicana 76: 1-45.

Ross H, Murugan S, Sibon W (2008) Testing the reliability of genetic methods of species identification via simulation. Systematic biology 57: 216-230.

Saarela JM, Sokoloff PC, Gillespie LJ, Consaul LL, Bull RD (2013) DNA barcoding the Canadian Arctic flora: core plastid barcodes (rbcL+ matK) for 490 vascular plant species. PloS One 8: e77982. DOI: 10.1371/journal.pone.0077982.

Sabás-Rosales JL, Siqueiros-Delgado ME, Valencia-Ávalos S, Enríquez-Enríquez ED (2017) Reconocimiento taxonómico de seis especies arbustivas de encinos (Quercus secc. Quercus: Fagaceae). Polibotánica 44: 11-38.

Saitou N, Nei M (1987) The neighbor-joining method: a new method for reconstructing phylogenetic trees. Molecular Biology and Evolution 4: 406-425.

Sharma R, Mahla HR, Mohapatra T, Bhargava SC, Sharma MM (2003) Isolating plant genomic DNA without liquid nitrogen. Plant Molecular Biology Reporter 21: 43-50. 
Simeone MC, Piredda R, Papini A, Vessella F, Schirone B (2013) Application of plastid and nuclear markers to DNA barcoding of Euro-Mediterranean oaks (Quercus, Fagaceae): problems, prospects and phylogenetic implications. Botanical Journal of the Linnean Society 172: 478-499.

Stecher G, Tamura K, Kumar S (2020) Molecular evolutionary genetics analysis (MEGA) for macOS. Molecular Biology and Evolution 37: 1237-1239.

Stoeckle MY, Gamble CC, Kirpekar R, Young G, Ahmed S, Little DP (2011) Commercial teas highlight plant DNA barcode identification successes and obstacles. Scientific Reports 1: 42. DOI: 10.1038/srep00042.

Tamura K, Peterson D, Peterson N, Stecher G, Nei M, Kumar S (2011) MEGA5: Molecular Evolutionary Genetics Analysis Using Maximum Likelihood, Evolutionary Distance, and Maximum Parsimony Methods. Molecular Biology and Evolution 28: 2731-2739.

Thompson JD, Higgins DG, Gibson TJ (1994) CLUSTAL W: improving the sensitivity of progressive multiple sequence alignment through sequence weighting, position-specific gap penalties and weight matrix choice. Nucleic Acids Research 22: 4673-4680.

Torres?Miranda A, Luna?Vega I, Oyama K (2011) Conservation biogeography of red oaks (Quercus, section Lobatae) in Mexico and Central America. American Journal of Botany 98: 290-305.

Valencia S (2004) Diversidad del género Quercus (Fagaceae) en México. Botanical Sciences 75: 33-53.

Valencia S, Rosales JLS, Arellano OJS (2016) A new species of Quercus, section Lobatae (Fagaceae) from the Sierra Madre Oriental, Mexico. Phytotaxa 269: 120-126.

Villarreal QJA, Encina DJA, Carranza PMA (2008) Los encinos (Quercus: Fagaceae) de Coahuila, México. Journal of the Botanical Research Institute of Texas 2: 1235-1278.

Yang J, Vázquez L, Chen X, Li H, Zhang H, Liu Z, Zhao G (2017) Development of chloroplast and nuclear DNA markers for Chinese oaks (Quercus subgenus Quercus) and assessment of their utility as DNA barcodes. Frontiers in Plant Science 8: 816. DOI: 10.3389 / fpls.2017.00816.

Yang Y, Zhou T, Duan D, Yang J, Feng L, Zhao G (2016) Comparative analysis of the complete chloroplast genomes of five Quercus species. Frontiers in Plant Science 7: 959. DOI: 10.3389 / fpls.2016.00959.

Yu J, Xue J, Zhou S (2011) New universal matK primers for DNA barcoding angiosperms. Journal of Systematics and Evolution 49: 176-181. 